\title{
Evidencia clínico epidemiológica de la existencia de síndrome pulmonar por hantavirus en Valdivia-Chile desde 1993
}

\author{
MARITZA NAVARRETE C. ${ }^{1}$, FRANCISCO SALDIAS N. ${ }^{2}$, M.V. RITA MANCILLA G. ${ }^{3}$, \\ T.M. LUIS ZAROR (Lic. Biol) ${ }^{1}$ y MARCELA FERRES G. ${ }^{4}$
}

\section{CLINICAL AND EPIDEMIOLOGICAL EVIDENCE OF HANTAVIRUS PULMONARY SYNDROME IN VALDIVIA-CHILE SINCE 1993}

The Hantavirus Pulmonary Syndrome (HPS) was described in USA in 1993. Cases of HPS have been retrospectively diagnosed by serological techniques back to 1959 in USA and to 1980 in Argentina. In Chile, cases have been reported since 1995. Infection with hantavirus was studied in three family groups who were attended at the Hospital Clínico Valdivia as probable severe cases of atypical pneumonia between February and April 1993. IgG anti hantavirus antibodies with titles $>1 / 6400$, were found in 5 of 7 patients. Their relatives were serologically negative. The patients presented different degrees of pulmonary and body damage, hypoxemia, thrombocytopenia and hypoalbuminemia. In some cases, increases in leukocyte counts, in GOT and LDH plasmatic levels and hemoconcentration, were found. The HPS related risk factors found were rurality, farm work surrounding their houses, contact with rodents and their excrements, cleaning of empty rural cottages and warehouses. The presence of HPS since 1993 was established in three family groups living in the province of Valdivia. These findings indicate that in unexplained cases of acute respiratory distress with epidemiological information of contact with rodents, it is necessary to bear the etiology of hantavirus in mind.

Key words: Hantavirus, Hantavirus Pulmonary Syndrome, Atypical pneumonia.

\footnotetext{
1 Instituto de Microbiología Clínica, Universidad Austral de Chile.

Servicio de Medicina, Hospital Clínico Valdivia

3 Departamento de Epidemiología, Servicio de Salud, Valdivia

4 Centro de Investigaciones Médicas, Pontificia Universidad Católica de Chile.
} 


\section{INTRODUCCION}

En E.U.A. se describió por primera vez durante 1993, un síndrome de dificultad respiratoria aguda en pacientes previamente sanos, caracterizado por un pródromo "gripal" con calofríos, cefalea, síntomas gastrointestinales, seguido del compromiso respiratorio rápidamente progresivo que requirió oxigenación suplementaria y que evolucionó como un síndrome de distress respiratorio tipo adulto (SDRA), letal en $52 \%$ de los casos. ${ }^{1}$ En la búsqueda de agentes etiológicos se identificó un nuevo virus de la Familia Bunyaviridae que pasó a pertenecer al género Hantavirus y al que se denominó Virus Sin Nombre (VSN). ${ }^{2,3}$ Su reservorio es un roedor silvestre, Peromyscus maniculatus (ratón venado), que se infecta con este virus en forma crónica y asintomática y lo excreta por orina, saliva y deposiciones. El hombre, huésped terminal y accidental en la cadena biológica, lo adquiere por inhalación de aerosoles de los fluidos que contienen el virus. El órgano más frecuentemente comprometido es el pulmón; sus pequeños vasos se dañan provocando una inundación del alvéolo que explica la sintomatología descrita. Su letalidad es alta (50 a 70\%), la recuperación y la inmunidad residual en aquellos que sobreviven es completa y de por vida. ${ }^{4}$

Cuando se identificó el agente causal del síndrome pulmonar por hantavirus (SPH) se desarrollaron técnicas de laboratorio que permitieron la búsqueda de anticuerpos específicos en el suero de los pacientes y técnicas de amplificación del genoma desde tejidos de biopsia y coágulo sanguíneo. Las técnicas serológicas así desarrolladas permitieron la identificación retrospectiva de casos de SPH en 1959 en E.U.A. ${ }^{5,6}$

En Sudamérica se han descrito brotes de SPH en Brasil, Paraguay y Argentina (década del '80). En Chile se han notificado casos aislados desde 1995, presentándose un brote en Coyhaique durante el mes de septiembre de 1997 que comprometió a dos familias, con una letalidad de $64 \% .^{8-10}$

El objetivo central del presente trabajo fue investigar retrospectivamente la infección por hantavirus en pacientes atendidos en el Hospital
Clínico Valdivia con diagnóstico de SDRA de etiología no precisada, mediante la búsqueda de anticuerpos específicos anti hantavirus. Secundariamente quisimos establecer la relación epidemiológica de los casos detectados con antecedentes de exposición a roedores, analizar su presentación clínica y parámetros de laboratorio y estudiar la presencia de infección antigua en los contactos de los casos índice, mediante técnicas serológicas para hantavirus.

\section{MATERIALES Y METODOS}

Se estudiaron retrospectivamente en 1998 tres grupos familiares atendidos en el Hospital Clínico Valdivia por sospecha diagnóstica de neumonía atípica grave-SDRA, entre el 6 de febrero y 23 de abril de 1993.

- grupo familiar I. Origen rural, compuesto por 3 miembros. El caso índice (padre) falleció antes de ser admitido a un centro hospitalario, luego enfermaron la esposa del fallecido y la hija de 3 años sobreviviendo ambas al episodio.

- grupo familiar II. Origen urbano, integrado por 9 miembros, 3 enfermaron. El caso índice (esposa) falleció, enfermando seguidamente el esposo y un hijo, ambos ingresados a una Unidad de Cuidados Intensivos (UCI). Una hija desarrolló un cuadro febril benigno que no requirió hospitalización. Los otros 5 miembros de la familia no presentaron sintomatología alguna.

- grupo familiar III. Origen rural, caso índice una mujer que enfermó bruscamente requiriendo hospitalización en UCI, pese a lo cual sobrevivió al episodio. Los 8 miembros restantes del grupo no presentaron enfermedad.

Estudio serológico específico para hantavirus

Se obtuvieron en 1998 sueros de los mencionados pacientes que sobrevivieron a un cuadro catalogado en ficha clínica como neumonía atípica- SDRA y de sus contactos, estudiándose con técnica de ELISA para IgM e IgG anti hantavirus. Para esto se localizó a los individuos escogidos a través de sus consultorios de referencia.

ELISA IgG antihantavirus: Se aplicó pri- 
mero el antígeno (VSN) y su control negativo sobre la fase sólida de una placa de ELISA de fondo en U de 96 pocillos. Se agregaron luego $42 \mu \mathrm{l}$ del suero del paciente en una dilución determinada y el sistema de detección constituido por anti-IgG humana conjugada con peroxidasa y el sustrato sulfonato de 2.2 '-azinodi-3-etil benztiazolina (ATBS).

ELISA IgM antihantavirus: Se utilizó una técnica de captura en fase sólida de una placa de ELISA de fondo en U de 96 pocillos. La IgM del paciente fue capturada con anti IgM humana cadena $\mu$. Luego se agregó antígeno específico de virus Laguna Negra y antigeno negativo anti - Laguna Negra preparado en conejo. El sistema de detección fue un anti $\mathrm{IgG}$ de conejo preparado en cabra, conjugado con peroxidasa y sustrato ATBS.

Tanto para detectar IgG como IgM, los sueros se corrieron en diluciones factor 4 desde $1 /$ 100 a 1/6.400. En cada prueba se utilizaron sueros de título conocido como control positivo y sueros controles negativos ${ }^{4,11}$

\section{Estudio de antecedentes epidemiologicos}

Se aplicó una encuesta epidemiológica a los miembros de los tres grupos familiares, utilizándose para estos efectos la encuesta elaborada por el Ministerio de Salud, (circular $\mathrm{N}^{\circ} 2 \mathrm{~A}$ / 37 del 8 de Octubre de 1997).

Esta ficha epidemiológica considera los siguientes puntos:

- Antecedentes del paciente. Estructura familiar

- Características de la vivienda, lugar de trabajo y saneamiento básico.

- Actividades desarrolladas en los últimos dos meses relacionadas con agricultura, limpieza y recreación.

- Situaciones específicas de contacto con roedores en el hogar, trabajo y actividad recreacional.

\section{Estudio de parámetros clínicos y de laboratorio}

Se revisó las fichas clínicas y se tabuló los datos clínicos y de laboratorio.

En los pacientes que estuvieron hospitalizados se buscaron dirigidamente los siguientes parámetros de laboratorio: radiografía de tórax frontal, gases arteriales respirando aire ambiente y en reposo, cultivo corriente de expectoración, hemocultivos, baciloscopias, hemograma completo con recuento de plaquetas y perfil bioquímico. En algunos de los pacientes se había efectuado un mayor estudio etiológico que incluyó: IFI para virus influenza y estudio serológico para Mycoplasma pneumoniae y Legionella pneumophila (IgM específica en ambos casos).

Las variables numéricas obtenidas fueron analizadas con promedios y desviaciones estándar.

\section{RESULTADOS}

\section{Perfil epidemiológico}

En la Tabla 1 se muestra la conformación de los grupos familiares, el perfil epidemiológico de los grupos en estudio y su relación con el caso índice. El Grupo I se conformó por 3 miembros de los cuales todos enfermaron, falleciendo uno de ellos; el Grupo II con un total de 9 miembros, de los cuales tres enfermaron, falleciendo uno; el grupo III formado por 9 personas de las cuales una enfermó, sobreviviendo al cuadro clínico.

En el grupo I el período de latencia entre el caso índice y los nuevos casos familiares fluctuó entre 3 y 53 días, en el segundo grupo fue de 4 a 70 días.

La tasa de infección clínica y/o seroconversión global fue de $33 \%$ (7/21), la letalidad por SPH fue de $28,6 \%(2 / 7)$.

\section{Estudio serológico}

Se detectó la presencia de anticuerpos antihantavirus del tipo IgG con títulos $>1 / 6.400$, en 5 de 7 pacientes con diagnóstico de neumonía atípica-SDRA, de los 2 restantes (fallecidos) no había muestras para análisis.

Se realizó el estudio para $\operatorname{IgM~e~IgG~en~todos~}$ los contactos del caso índice: en el grupo familiar II se estudiaron 5 contactos, de los dos restantes no fue posible obtener muestras; en el grupo III se estudiaron los 8 contactos; en el grupo familiar I no hubo contactos a estudiar. En todos los contactos la serología resultó negativa. 
Tabla 1. Perfil epidemiológico de los grupos familiares y su relación con el caso índice

\begin{tabular}{|c|c|c|c|c|c|c|c|}
\hline Sexo & $\begin{array}{l}\text { Edad } \\
\text { (años) }\end{array}$ & Parentesco & $\begin{array}{l}\text { Pródromos } \\
\text { (días) }\end{array}$ & $\begin{array}{l}\text { Latencia } \\
\text { (días) }\end{array}$ & $\begin{array}{l}\text { Hospitali- } \\
\text { zación }\end{array}$ & IgG* & Resolución \\
\hline \multicolumn{8}{|c|}{ Grupo familiar I } \\
\hline M & 37 & Padre (índice) & 7 & no & no & $\begin{array}{c}\text { Sin } \\
\text { muestra }\end{array}$ & $\dagger$ \\
\hline $\mathrm{F}$ & 24 & Madre & 7 & 30 & UCI & + & Vivo \\
\hline $\mathrm{F}$ & 3 & Hija & 4 & 53 & UCI & + & Vivo \\
\hline \multicolumn{8}{|c|}{ Grupo familiar II } \\
\hline $\mathrm{F}$ & 54 & Madre (índice) & 5 & no & UCI & $\begin{array}{c}\text { Sin } \\
\text { muestra }\end{array}$ & $\dagger$ \\
\hline $\mathrm{F}$ & 15 & Hija & 7 & 04 & no & - & Vivo \\
\hline M & 59 & Padre & 7 & 21 & UCI & + & Vivo \\
\hline M & 30 & Hijo & 8 & 70 & UCI & + & Vivo \\
\hline M & 32 & Hijo & - & - & - & - & $\mathrm{S} / \mathrm{I}$ \\
\hline $\mathrm{F}$ & 22 & Hija & - & - & - & - & $\mathrm{S} / \mathrm{I}$ \\
\hline M & 3 & Nieto & - & - & - & $\begin{array}{c}\text { Sin } \\
\text { muestra }\end{array}$ & S/enfermedad \\
\hline $\mathrm{F}$ & 26 & Nuera & - & - & - & $\begin{array}{c}\text { Sin } \\
\text { muestra }\end{array}$ & S/enfermedad \\
\hline $\mathrm{F}$ & & Sin & - & - & - & - & $\mathrm{S} / \mathrm{I}$ \\
\hline \multicolumn{8}{|c|}{ Grupo familiar III } \\
\hline $\mathrm{F}$ & 20 & Hija (índice) & 3 & no & UCI & + & Vivo \\
\hline M & 50 & Padre & - & - & - & - & $\mathrm{S} / \mathrm{I}$ \\
\hline $\mathrm{F}$ & 39 & Madre & - & - & - & - & $\mathrm{S} / \mathrm{I}$ \\
\hline $4 \mathrm{M} / 2 \mathrm{~F}$ & $11-21$ & 6 hermanos & - & - & - & - & $\mathrm{S} / \mathrm{I}$ \\
\hline
\end{tabular}

\section{Aspectos clínicos}

El estudio de las historias clínicas de los 6 pacientes con infección por hantavirus (un paciente falleció antes de ser admitido en un centro hospitalario), mostró una forma clínica con diversos grados de compromiso pulmonar y sistémico, con una letalidad de 28,6\%. Sólo 2 de ellos necesitaron ventilación mecánica aunque todos recibieron oxigenación suplementaria por al menos cuatro días.

\section{Laboratorio}

Los exámenes de laboratorio más relevantes y compatibles con el diagnóstico de SPH fueron hipoxemia, trombocitopenia, hipoproteine-mia e hipoalbuminemia en todos los pacientes, observándose en algunos de ellos leucocitosis, hemoconcentración, elevación de GOT y DHL (Tabla 2).

Los estudios etiológicos para virus influenza, M. pneumoniae y L. pneumophila en los pacientes con síntomas respiratorios fueron negativos o sin seroconversión.

\section{Antecedentes epidemiológicos medioambientales}

Las actividades desarrolladas al momento de la infección por los grupos familiares en estudio fueron: en los grupos I y III actividades propias de la ruralidad; los miembros del grupo familiar 
Tabla 2. Síndrome pulmonar por hantavirus. Exámenes de laboratorio. (Valores plasmáticos promedio y desviación estándar)

\begin{tabular}{|c|c|}
\hline Exámenes & Resultados (DS) \\
\hline $\mathrm{PaO}_{2} \mathrm{mmHg}$ & $42 \quad( \pm 12)$ \\
\hline $\mathrm{PaCO}_{2}^{2} \mathrm{mmHg}$ & $27 \quad( \pm 4,7)$ \\
\hline Hematocrito $\%$ & $49,9( \pm 8,6)$ \\
\hline Leucocitos $/ \mathrm{mm}^{3}$ & $21.230 \quad( \pm 15.900)$ \\
\hline Plaquetas $/ \mathrm{mm}^{3}$ & $51.500 \quad( \pm 16.900)$ \\
\hline Proteínas gr/l & $5,4( \pm 0,7)$ \\
\hline Albúmina gr/l & $2,6( \pm 0,47)$ \\
\hline Calcio mg\% & $6,8( \pm 0,84)$ \\
\hline GOT U/l & $218 \quad( \pm 117)$ \\
\hline DHL U/l & $743 \quad( \pm 552)$ \\
\hline Colesterol mg\% & $106( \pm 12)$ \\
\hline Sodio meq/l & $129 \quad( \pm 8,6)$ \\
\hline Potasio meq/l & $3,8( \pm 0,47)$ \\
\hline
\end{tabular}

II, residentes urbanos, realizaban actividades recreativas en el sector costero.

Los factores de riesgo a los cuales se expusieron los grupos familiares se resumen en la Tabla 3. El grupo familiar I presentó como factores de riesgo la ruralidad, la realización de trabajos agrícolas en el hogar y el contacto con roedores y sus excreciones. En el grupo familiar II los factores de exposición estuvieron relacio- nados con limpieza de cabaña previamente desocupada en el sector rural. El caso índice del grupo familiar III realizó limpieza de una bodega, trabajo agrícola en torno al domicilio (ambos en el sector rural), y contacto con fecas de roedores, especialmente en la bodega en la cual se realizó limpieza.

Los miembros de los grupos familiares I y II que enfermaron en contacto con el caso índice, compartieron los mismos factores de riesgo que éste, por lo cual no se puede considerar la transmisión de persona a persona como probable forma de contagio.

Saneamiento básico. Las viviendas de los grupos familiares I y III no disponían de alcantarillado ni agua potable. En el grupo familiar I1 se sumaba una mala disposición de basuras intra y extra domiciliaria y casa en pésimas condiciones estructurales. La cabaña de veraneo en que se infectó el grupo familiar II era estructuralmente buena, pero con varias entradas por las que podían introducirse roedores pequeños. El sector involucrado en el brote está conformado por cerros pertenecientes a la cordillera de la costa, en la ribera norte del río Valdivia y al este de la bahía de Corral. En la ladera oeste de los cerros, bordeando el río Valdivia, se ubican casas dispersas donde resi-

Tabla 3. Factores de riesgo asociados al contagio por hantavirus en tres grupos familiares con casos de SPH. Valdivia 1993

\begin{tabular}{lccc}
\hline Factores de riesgo & Grupo familiar I & Grupo familiar II & Grupo familiar III \\
\hline $\begin{array}{l}\text { Ruralidad } \\
\text { Realización de trabajos }\end{array}$ & + & + & + \\
agrícolas & + & - & + \\
$\begin{array}{l}\text { Limpieza de cabañas o } \\
\text { bodegas desocupadas } \\
\text { Contacto con roedores } \\
\text { vivos o muertos }\end{array}$ & - & + & + \\
$\begin{array}{l}\text { Contacto con fecas } \\
\text { de roedores }\end{array}$ & + & - & - \\
$\begin{array}{l}\text { Contacto con alimentos } \\
\text { roídos } \\
\text { Contacto con persona } \\
\text { afectada por SPH } \\
\begin{array}{l}\text { Contacto con sangre o fluidos } \\
\text { corporales de personas con SPH }\end{array}\end{array}$ & + & - & + \\
Heridas provocadas \\
por roedores
\end{tabular}


den especialmente pescadores artesanales, aquí se presentaron los casos que pertenecen a los grupos familiares I y III, existe además, por el sector costero frente a la bahía, un poblado rural destinada a balneario, lugar donde se produjeron los casos pertenecientes al grupo familiar II.

\section{DISCUSION}

El género Hantavirus incluye un grupo de virus cuyo reservorio natural lo constituyen los roedores silvestres. Aunque estos virus producen infección asintomática en los roedores, ellos pueden causar enfermedad en humanos con manifestaciones leves tales como fiebre y mialgias, hasta un síndrome hemorrágico con shock, insuficiencia renal y muerte. El reciente conocimiento del SPH ha cambiado dramáticamente nuestro entendimiento del espectro clínico y epidemiológico de las infecciones por hantavirus.

En Chile se notificó el primer caso clínico de SPH en 1995, existiendo a la fecha un total de 118 casos confirmados ${ }^{12}$; sin embargo, por el desarrollo de técnicas de diagnóstico serológico, histopatológicas y de biología molecular, se ha podido establecer en forma cierta que la infección se ha encontrado presente en nuestro continente por al menos cuatro décadas (1959 en E.U.A., ${ }^{5}$ década de los 80 en Argentina) ${ }^{4}$. Con este reporte en la provincia de Valdivia Chile, de la ocurrencia de un brote que afectó a tres grupos familiares, catalogados en esa fecha como casos de neumonía atípica y SDRA, confirmamos la existencia de SPH en nuestro país desde al menos 1993. Recientemente se ha presentado un nuevo caso de SPH en el área estudiada, afectando a un paciente preescolar, lo cual ha motivado un próximo estudio de reservorio en el sector costero de Valdivia. ${ }^{13}$

Los pacientes estudiados con diagnóstico de neumonía atípica y SDRA mostraron un cuadro clínico semejante al descrito en E.U.A. a partir de 1993, con compromiso pulmonar de magnitud variable, requiriendo ventilación mecánica dos de ellos y el resto, oxigenación suplementaria. Llama la atención, la baja letalidad de 28,6\% en contraste con la descrita en los brotes de
E.U.A. de $52 \%$, y con la letalidad actual en Chile que alcanza a 50,0\%. ${ }^{12}$ En la exploración de laboratorio efectuada a estos casos aparecen los signos clásicos sugerentes de SPH: hemoconcentración, leucocitosis, plaquetopenia, ascenso de enzimas hepáticas y de DHL. ${ }^{14}$

$\mathrm{La}$ IgG específica anti hantavirus se mantuvo elevada con títulos > 1/6.400 aún tras 5 años de ocurrido el cuadro clínico. En un estudio retrospectivo de infecciones clínicas por hantavirus en Argentina se detectaron anticuerpos antihantavirus hasta 110 días tras el cuadro de SPH, período que duró el seguimiento serológico en esa experiencia. ${ }^{4}$

$\mathrm{Al}$ analizar los antecedentes epidemiológicos de los grupos familiares, se ve una clara relación con la exposición a roedores silvestres; sin embargo, en el caso del grupo familiar II es posible plantear la transmisión persona a persona -situación que fuera documentada epidemiológicamente en un brote de SPH ocurrido en Argentina en 1996- dado el período de latencia de 70 días en uno de los casos clínicos confirmados serológicamente y en consideración a que el período de incubación en este caso sobrepasaría el mayor descrito para el SPH, aproximadamente de 42 días. ${ }^{16-17}$

Existen evidencias clínicas y anátomo patológicas y de laboratorio suficientes para catalogar a los dos casos fallecidos como típicos de $\mathrm{SPH}$, en el caso índice del grupo familiar I por elementos tales como síndrome gripal, dificultad respiratoria, cianosis, muerte súbita y tipo de compromiso pulmonar en la anatomía patológica, y en el segundo caso índice, por el compromiso respiratorio progresivo con hipoxemia severa, trombocitopenia, leucocitosis con desviación a izquierda y hemoconcentración.

Desde el punto de vista ecológico, las características generales de la vegetación en el área involucrada en el brote son ideales para la existencia de roedores silvestres como Oligoryzomys longicaudatus y Abrothrix olivaceus. ${ }^{18}$ La vegetación de los cerros está constituida por bosque nativo tipo hidrófilo templado, "bosque valdiviano". En las partes más bajas se encuentran sectores intervenidos por el hombre con talado total e invadido especialmente por espinillo (Ulex $s p$.); existen además matorrales de zarzamora (Rubus sp.), maqui 
(Aristotelia chilensis) y quila (Chusquea sp.). ${ }^{19}$ La cobertura de los matorrales es de aproximadamente $60 \%$, lo que da protección y abrigo a especies de roedores silvestres del área como $O$. longicaudatus, A. olivaceus, Abrothrix longipilis y Loxodontomys micropus. ${ }^{20-21}$

Las especies de roedores $O$. longicaudatus, A. olivaceus y A. longipilis han resultado positivas a las pruebas serológicas a hantavirus, siendo $O$. longicaudatus el huésped primario provisional de virus Andes. Tanto en $O$. longicaudatus como en A. olivaceus se encontraron anticuerpos contra hantavirus en el estudio de reservorios realizado en el sur de Chile. ${ }^{22}$ A las características de vegetación favorables para la existencia de ratones silvestres, se suman las construcciones de las viviendas y construcciones anexas desprotegidas a la entrada de roedores, escaso conocimiento sobre el tema en esa época y además, el acontecimiento que en 1993 nos encontrábamos en el Sur de Chile, sufriendo un fenómeno de ratada producto del florecimiento de la quila, todo lo cual contribuyó a que se dieran las condiciones propicias para la presentación de estos casos. ${ }^{23}$

Aunque sólo recientemente se reconoce a hantavirus como el agente causal del SPH en E.U.A. y Latinoamérica, es posible que estos agentes hayan estado con nosotros por muchos años y los cambios en las condiciones climáticas hayan permitido un aumento de la población de roedores con el consiguiente aumento del contacto de estos roedores con humanos. Esta sería la razón de la aparente emergencia de esta infección que verdaderamente ha convivido con nosotros desde antes de su mejor conocimiento logrado gracias al desarrollo de las técnicas diagnósticas actuales. ${ }^{24}$

El mayor aporte de este trabajo reside en la evidencia de infección por hantavirus en pacientes con diagnóstico de neumonía atípica y SDRA de etiología no precisada. A partir de estos hallazgos en la provincia de Valdivia, es necesario tener en cuenta la probable etiología por hantavirus en todos los futuros casos inexplicados de SDRA o ante los casos de edema pulmonar agudo no cardiogénico, asociados a antecedentes epidemiológicos sugerentes de un posible contacto con roedores silvestres. Así también, ciertos signos como taquipnea, hemoconcentración, trombocitopenia, leucocitosis con desviación a izquierda pueden orientar en el diagnóstico. ${ }^{5,25}$

Así mismo, se abren nuevas preguntas como identificación del roedor involucrado en el brote de 1993 y porcentaje de humanos infectados en el sector rural costero donde ocurrió este brote. Nuevos y emergentes patógenos presentan y presentarán desafíos a clínicos, infectólogos, microbiólogos y biólogos moleculares.

\section{RESUMEN}

El síndrome pulmonar por hantavirus (SPH) fue descrito en E.U.A. en 1993. Se notificaron casos en Chile desde 1995. Utilizando serología se han identificado retrospectivamente casos desde 1959 en E.U.A. y 1980 en Argentina.

En tres grupos familiares atendidos en el Hospital Regional Valdivia entre febrero y abril de 1993 se encontraron, en un estudio retrospectivo efectuado 5 años más tarde, anticuerpos IgG anti-hantavirus con títulos > 1/6.400, en 5 de 7 pacientes atendidos con el diagnóstico de neumonía atípica - síndrome de distress respiratorio tipo adulto (SDRA) . Sus contactos presentaron serología negativa. El cuadro clínico de estos pacientes se caracterizó por diversos grados de compromiso pulmonar y sistémico, hipoxemia, plaquetopenia, hipoproteinemia e hipoalbuminemia; en algunos, leucocitosis, hemoconcentración, GOT y DHL elevadas. De los factores de riesgo reconocidos para adquirir $\mathrm{SPH}$, encontramos en esta serie: ruralidad, trabajo agrícola en la vecindad del domicilio, contacto con roedores y sus excreciones, limpieza de cabaña o bodegas desocupadas.

Se establece retrospectivamente la presencia del SPH en la provincia de Valdivia a partir de 1993 en tres grupos familiares. Los hallazgos hacen necesario tener presente la etiología de hantavirus en casos inexplicados de SDRA con el antecedente epidemiológico de contacto con roedores silvestres.

\section{BIBLIOGRAFIA}

1.- KHAN A, KHABBAZ R F, ARMSTRONG L et al. Hantavirus Pulmonary Syndrome: the first 100 US cases. J Infect Dis 1996; 173: 1297-303.

2.- BUTLER J, PETERS C J. Hantaviruses and 
Hantavirus Pulmonary Syndrome. Clin Infect Dis 1994; 19: 387-95.

3.- PETERS C J, KHAN A, ZAKI S. Hantaviruses in the United States. Arch Intern Med 1996; 156 (8): 705-6.

4.- PARISI M N, ENRIA D, PINI N, SABATTINI M. Detección retrospectiva de infecciones clínicas por hantavirus en la Argentina. Medicina (Buenos Aires) 1996; 5: 1-13.

5.- SHEFER A, TAPPERO J, BESSEC $J$ et al. Hantavirus Pulmonary Syndrome in California: Report of two cases and investigation. Clin Infect Dis 1994; 19: 1105-9.

6.- FRAMPTON J W, LANSER S, NICHOLS C R, ETTESTAD P J. Sin nombre virus infection in 1959. Lancet 1995; 346: 781-2.

7.- ENRIA D. Emergencia de los hantavirus en las Américas y en la Argentina. Medicina (Buenos Aires) 1998; 58 (Supl. I): 15-8.

8.- ESPINOZA R, VIAL P, NORIEGA L et al. Hantavirus Pulmonary Syndrome in a Chilean patient with recent travel in Bolivia. Emerg Infect Dis 1998; 4 (1): 93-5.

9.- VASCONCELOS M I, IVERSSON L B, DA ROSA A P A et al. Hantavirus Pulmonary Syndrome in the rural area of Juquitiba, São Paulo Metropolitan area, Brazil. Rev Inst Med Trop S Paulo 1997; 39 (4): 237-8.

10.- TAPIA G M. Síndrome pulmonar por hantavirus. Rev Chil Enf Respir 1997; 13: 103-10.

11.- KHAN A, KSIAZEK T, ZAKI S et al. Fatal Hantavirus Pulmonary Syndrome in an adolescent. Experience and Reason, 1994, 276-80.

12.- MINISTERIO DE SALUD DE CHILE. Hantavirus: Situación epidemiológica. http: //disap.minsal.cl/ epidemiología/enftrans/hanta.htlm

13.- WENZEL M, NAVARRETE M, ALBORNOZ C, RIOS R. Síndrome Pulmonar por Hantavirus en un preescolar de Valdivia, Chile. Libro resúmenes XV Congreso Chileno de Infectología, Agosto 1998, Santiago, Chile.

14.- CHILDS J, ROLLIN P. Emergence of Hantavirus Disease in the USA and Europe. Current Opinion Infect Dis 1994; 7: 220-4.

15.- ENRIA D, PADULA P, SEGURA E et al. Hantavirus pulmonary syndrome in Argentina. Possibility of person to person transmission. Medicina (Buenos Aires), 1996; 56: 709-11.

16.- PADULA P, EDELSTEIN A, MIGUEL S, LOPEZ $\mathrm{N}$, ROSSI C, RABINOBICH R. Brote epidémico del Síndrome Pulmonar por Hantavirus en la Argentina, Evidencia molecular de la transmisión de persona a persona del virus Andes. Medicina (Buenos Aires) 1998 ; 58 (Supl.I) : 27-36.

17.- WELLS R M, SOSA S, YADON Z E et al and the Hantavirus Pulmonary Syndrome Study Group for Patagonia. Unusual hantavirus outbreak in southern Argentina: person to person transmission? Emerg Infect Dis 1997; 3: 171-4.

18.- MURUA R, GONZALEZ L A. Microhabitat selection in two chilean cricetid rodents. Oecologia 1982; 52: 12-5.

19.- GAJARDO R. La vegetación natural de Chile, Clasificación y distribución geográfica. 1995; Editorial Universitaria. Santiago.

20.- MURUA R. Comunidades de mamíferos del bosque templado de Chile. En Armesto, J.J., Villagrán C \& Kalin M. eds. Ecología de los bosques nativos de Chile. Editorial Universitaria, Santiago, 1996; pp: 113-33.

21.- MURUA R. Ecología de los reservorios silvestres de hantavirus en Chile. Rev Chil Infect 1998; 15 (2): 79-83.

22.- MILLS J, CHILDS J, KSIZEK T, PETERS C J, VELLECA W. Métodos para trampeo y muestreo de pequeños mamíferos para estudios virológicos. Washington, 1998. OPS/HPC/HCT. 104:41-44.

23.- MURUA R, GONZALEZ L A, GONZALEZ M, JOFRE C. Efectos del florecimiento del arbusto Chusquea quila, Kunth (Poacea) sobre la demografía de poblaciones de roedores de los bosques templados fríos del sur de Chile. Bol Soc Biol Concepción 1996; 67: 37-42.

24.- SCHMALJOHN C, HJELLE B. Hantaviruses: A global disease problem. Emerg Infect Dis 1997; 3 (2): 95-102.

25.- THORTON G. General infectious disease: new and emerging pathogens. Current Opinion Infect Dis 1994; 7: 219.

Correspondencia a:

Maritza Navarrete C

Fax 56 (63) 293300

E-mail: farmacia@uach.cl 